'Departamento de Radioterapia Oncológica, Clínica Instituto de Radiomedicina (IRAM), Santiago, Chile.

2Programa de Radioterapia Oncológica. Facultad de

Medicina. Universidad Diego Portales. Santiago, Chile.

Fuente de apoyo financiero: Ninguno.

Recibido el 12 de mayo de 2017 aceptado el 18 de octubre de 2017.

Correspondencia a: Andrés Vargas Avenida Américo Vespucio 1314, Vitacura, Santiago. avargas@iram.cl

\section{Sarcoma de Kaposi: variedades clínicas y resultados del tratamiento local con radioterapia}

\author{
ANDRÉS VARGAS ${ }^{1}$, SEBASTIÁN SOLÉ ${ }^{1,2}$
}

\section{Local radiation treatment of Kaposi's sarcoma. Clinical varieties and treatment outcomes}

Background: Kaposi's sarcoma (KS) is a rare tumor that affects mainly immunodeficient and elderly patients. Aim: To describe the features and outcomes of a cohort of patients with different types of KS treated locally with radiotherapy (RT). Patients and Methods: Retrospective review of patients with KS referred to our radiation oncology center for local treatment of symptomatic lesions that did not respond to high activity anti-retroviral therapy or chemotherapy. Results: Between January 1995 and December 2016, 20 patients with a median age of 40 years (18 males) with KS were assessed and treated with $R T$ due to symptomatic lesions. The most common KS type was epidemic in 15 patients, followed by the classic type in three and the iatrogenic variety in two. In patients with non-epidemic varieties, SK was exclusively cutaneous, while visceral involvement was present in $46 \%$ of patients with epidemic SK. Complete response was observed in 95\% of cases. Skin toxicity caused by RT was mild in all cases. Conclusions: RT may be considered as a reasonable local treatment choice in classic, iatrogenic and epidemic varieties of $K S$.

(Rev Med Chile 2018; 146: 32-38)

Key words: Radiotherapy; Sarcoma, Kaposi; Therapeutics.
4 1 sarcoma de Kaposi es una angiomatosis sistémica que se manifiesta primariamente como nódulos vasculares múltiples en la piel, pudiendo también afectar a las mucosas de los tractos aéreo o digestivo, linfonodos y órganos sólidos. Se han descrito cuatro variedades, cada una de ellas con particulares características epidemiológicas, clínicas y terapéuticas: clásica, epidémica asociada a SIDA, iatrogénica y endémica africana.

La forma clásica se manifiesta principalmente en población de origen judío, de Europa del este y mediterránea, aparece hacia la séptima década de la vida y afecta principalmente a hombres ${ }^{1}$. Frecuentemente se localiza a nivel de extremidades inferiores. Tiende a ser una condición crónica, por lo que los pacientes pueden alcanzan largas supervivencias.
La forma epidémica, asociada al SIDA, se manifiesta con afectación de piel o extracutánea, ya sea gastrointestinal, pulmonar, ganglionar $o$ múltiple. La afectación ósea es infrecuente ${ }^{2}$.

La forma iatrogénica es propia de pacientes en tratamiento inmunosupresor secundario a trasplantes o para el control de enfermedades sistémicas de causa autoinmune ${ }^{3}$.

Finalmente, la forma endémica africana afecta principalmente a hombres, puede tener un curso indolente o agresivo y se presenta con lesiones de morfología diversa ${ }^{4}$.

En Chile existen escasos datos oficiales acerca de la prevalencia del SK y respecto de sus diferentes variedades ${ }^{5,6}$. Adicionalmente, sus características clínicas y la experiencia en su manejo específico han sido escasamente comunicadas ${ }^{7-11}$. 
Habitualmente, el SK tiene una distribución multifocal al momento del diagnóstico, en este contexto, la terapia antirretroviral de alto grado de actividad (TAAGA), con o sin quimioterapia (QT) asociada suele ser el tratamiento de entrada ${ }^{8,9}$.

La radioterapia (RT) es un tratamiento eficaz para el tratamiento local del SK. La literatura disponible da cuenta de su efectividad, tanto en la curación como en la paliación de lesiones que cursan con hemorragia, edema o dolor ${ }^{10,11}$. Además, se han reportado excelentes resultados cosméticos con esta modalidad de tratamiento ${ }^{12,13}$.

En este estudio se describen las características clínicas y los resultados del tratamiento con RT en una cohorte retrospectiva de pacientes con SK asistidos en nuestro centro en las últimas dos décadas.

\section{Pacientes y Métodos}

Mediante los registros de pacientes referidos a nuestro centro, se identificó a todos aquellos derivados con el diagnóstico de SK desde enero de 1995 hasta diciembre de $2016(n=26)$.

La derivación se realizó desde un comité oncológico $(\mathrm{n}=20)$ o por interconsulta médica desde una consulta privada por un especialista no oncólogo $(n=6)$. De entre los pacientes identificados, 6 pacientes no recibieron RT debido a que se encontraban asintomáticos $(\mathrm{n}=4)$ o por propia decisión $(\mathrm{n}=2)$.

En los 20 pacientes tratados, se realizó tratamiento con la intención de controlar síntomas como edema, sangrado o dolor. Se registraron variables sociodemográficas como sexo y edad, así como variables clínicas: presencia o no de inmunosupresión, causa de la inmunosupresión, extensión de la enfermedad (cutánea versus cutáneo-visceral), localización anatómica (cabeza/ cuello, tronco, extremidades, mucosa anal/oral, ganglionar, óseo) y tratamiento sistémico previo a la RT (TAAGA, QT o ambos).

En todos los pacientes se registró la dosis de $\mathrm{RT}$, la manifestación de efectos adversos locales secundarios al tratamiento según los criterios Radiation Therapy Oncology Group y European Organization for Research and Treatment of Cancer (RTOG/EORTC $)^{18}$, la respuesta clínica al mes post tratamiento y la presencia o no de recidivas locales, así como la progresión a otros niveles durante el seguimiento.
Previo al inicio del tratamiento, todos los pacientes incluidos en este estudio habían firmado un consentimiento informado autorizando el uso de sus datos clínicos para fines de investigación.

Las características basales de los pacientes incluidos se reportaron como números absolutos y porcentajes. La comparación de las variables categóricas entre grupos se realizó utilizando el test de $\chi^{2}$ o el test exacto de Fisher, según fuese apropiado. Todos los tests estadísticos fueron bilaterales y la significación estadística fue definida como $\mathrm{p}<0,05$. El análisis estadístico se realizó mediante el programa SPSS versión 20 .

\section{Resultados}

Los datos demográficos y las características clínicas generales de los pacientes incluidos en este estudio se muestran en la Tabla 1. La mediana de seguimiento fue 10,5 meses (rango 2-120 meses).

\section{Tabla 1. Datos demográficos y características clínicas generales de los pacientes incluidos en este estudio}

\begin{tabular}{|lr|}
\hline Sexo (\%) & \\
- Hombre & $18(90 \%)$ \\
- Mujer & $2(10 \%)$ \\
\hline Edad (mediana) & 40,5 años \\
Variedad (\%) & \\
- Epidémica & $15(75 \%)$ \\
- Endémica & $3(15 \%)$ \\
- latrogénica & $2(10 \%)$ \\
Síntoma & \\
- Dolor & 16 \\
- Edema local & 7 \\
- Hemorragia local & 5 \\
Estadio & \\
- Localizado & $13(65 \%)$ \\
- Cutáneo-visceral & $7(35 \%)$ \\
Localización & \\
- Extremidades & $10(50 \%)$ \\
- Mucosa oral/anal & $4(20 \%)$ \\
- Tronco & $2(10 \%)$ \\
- Óseo & $2(10 \%)$ \\
- Piel de cabeza/ cuello & $1(5 \%)$ \\
- Ganglionar & $1(5 \%)$ \\
\hline
\end{tabular}


La variedad más frecuente de SK fue la epidémica, presente en 75\% de los pacientes. El 15\% de los pacientes presentó la variedad clásica y $10 \%$ la variante iatrogénica. Globalmente, $40 \%$ de los pacientes presentaba más de un síntoma al momento del tratamiento con RT.

Entre los pacientes con SK clásico $(\mathrm{n}=3)$, todos tenían ascendencia europea directa y en todos ellos la afectación fue exclusivamente cutánea. La edad promedio al momento del SK fue 75,3 años. Dado que no presentaban una afectación cutánea difusa ni compromiso visceral, no recibieron tratamiento con QT. Las dosis de 30 Gy/10 fracciones $(n=2)$ y 60 Gy/30 fracciones $(\mathrm{n}=1)$ fueron efectivas para alcanzar la respuesta clínica completa y el alivio sintomático en los tres pacientes. Un paciente presentó epitelitis grado 1. El seguimiento promedio en este grupo fue de 29 meses (60, 11 y 16 meses), tiempo durante el cual ningún paciente presentó recidiva local.
De los pacientes con SK iatrogénico $(\mathrm{n}=2)$, un paciente se encontraba en tratamiento inmunosupresor con prednisona y tacrolimus debido a un trasplante hepático, mientras que el segundo paciente estaba en tratamiento con prednisona y sulfazalacina debido a una artritis reumatoide. El promedio de edad de estos pacientes al momento del diagnóstico de SK fue 65,5 años. Ningún paciente presentó afectación cutánea difusa ni visceral, por lo que no recibieron tratamiento con QT. En ninguno de ellos se ajustó o cambió el tratamiento inmunosupresor. Los esquemas de tratamiento utilizados fueron 40 Gy en 20 fracciones $(n=1)$ y 30 Gy en 10 fracciones $(n=1)$, ambos pacientes presentaron una respuesta clínica completa. Un paciente presentó epitelitis grado 2. El paciente trasplantado no presentó recurrencia local a los 12 meses de seguimiento, sin embargo, evolucionó con progresión en otras zonas, por lo que recibió nuevamente $\mathrm{RT}$ en dos ocasiones, con

Tabla 2. Variables de tratamiento para los tres tipos de SK incluidos en el presente estudio

\begin{tabular}{|c|c|c|c|}
\hline & Epidémico (n = 15) & Clásico (n = 3) & latrogénico $(n=2)$ \\
\hline \multicolumn{4}{|l|}{ Extensión } \\
\hline Localizado & 8 & 3 & 2 \\
\hline Cutáneo-visceral & 7 & 0 & 0 \\
\hline \multicolumn{4}{|l|}{ Tratamiento sistémico } \\
\hline TAAGA & 13 & - & - \\
\hline QT & 5 & 0 & 0 \\
\hline \multicolumn{4}{|l|}{ Dosis de RT } \\
\hline 60 Gy/30 fracciones & 0 & 1 & 0 \\
\hline 40 Gy/20 fracciones & 1 & 0 & 1 \\
\hline $30 \mathrm{~Gy} / 10$ fracciones & 3 & 2 & 1 \\
\hline 20 Gy/4 fracciones & 7 & 0 & 0 \\
\hline 8 Gy/1 fracción & 4 & 0 & 0 \\
\hline \multicolumn{4}{|l|}{ Respuesta a la RT } \\
\hline $\mathrm{RC}$ & 12 & 3 & 2 \\
\hline $\mathrm{RP}$ & 2 & 0 & 0 \\
\hline NR & 1 & 0 & 0 \\
\hline \multicolumn{4}{|l|}{ Tolerancia a la RT } \\
\hline Sin toxicidad aguda & 10 & 2 & 1 \\
\hline Toxicidad grado 1-2 & 5 & 1 & 1 \\
\hline Toxicidad grado $\geq 3$ & 0 & 0 & 0 \\
\hline Recidiva local & 0 & 0 & 0 \\
\hline Progresión a otros niveles & 7 & 0 & 1 \\
\hline
\end{tabular}

TAAGA: Terapia antirretroviral de alto grado de actividad; QT: quimioterapia; RC: respuesta completa; RP: respuesta parcial; NR: no respuesta. 
respuesta completa de las lesiones y sin toxicidad asociada. El segundo paciente de este grupo fue seguido durante 9 meses, tiempo durante el cual no se registró recurrencia.

En pacientes con SK epidémico $(\mathrm{n}=15)$, la afectación cutáneo-visceral fue más frecuente que en las variedades no asociadas a VIH (46\%). En 2 pacientes se evidenció compromiso visceral asociado a afectación ósea extensa y sintomática, mientras que en 1 paciente la afectación cutánea se asoció a compromiso ganglionar. En 4/15 pacientes $(26,6 \%)$ el diagnóstico de SK se realizó sincrónicamente al de VIH. El 86,6\% de los pacientes con SK epidémico se encontraba en tratamiento con TAAGA y solo 33,3\% había recibido tratamiento con QT al momento de la derivación a RT. En cuanto al estatus inmunológico, 8/15 pacientes tenían un nivel de CD $4<500$ y carga viral detectable.

Los pacientes con lesiones óseas y ganglionares fueron tratados con intención paliativa con una dosis de 20 Gy en 4 fracciones, todos ellos habían presentado una escasa respuesta al tratamiento con TAAGA y QT. Tras el tratamiento con RT, en cuatro pacientes con lesiones de piel se evidenció epitelitis grado 1 y un paciente presentó epitelitis grado 2 .

En suma, el único efecto adverso agudo en los tres grupos fue la epitelitis, presente en 7/20 pacientes (35\%), en todos los casos fue leve o moderada (grado 1 o 2). La toxicidad del tratamiento con RT fue similar entre el grupo con SK epidémico y aquellos afectos por variantes no epidémicas.

En la Tabla 2 se resumen las variables de tratamiento para los tres tipos de SK incluidos en el presente estudio.

Durante el seguimiento no se documentaron recidivas locales en piel ni progresión clínica de lesiones ganglionares u óseas tratadas. La progresión de la enfermedad en otras zonas anatómicas fue más frecuente en la variedad epidémica que en las no asociadas a VIH (46,6\% versus $20 \%$ de pacientes respectivamente, $\mathrm{p}=0,22)$.

\section{Discusión}

Según datos publicados, el SK es una neoplasia infrecuente en Chile. Lasso et al. reportaron una incidencia de la variedad epidémica que osciló entre 0,47 casos por 100 personas/año (1996) y 1,52 casos por 100 personas/año (1999) ${ }^{8}$. Los datos más recientemente publicados en Chile son los registrados en el quinquenio 2003-2007, para dicho periodo, la tasa bruta de incidencia fue de 0,2 casos por 100.000 personas $/ \mathrm{año}^{5}$. Aun cuando en el presente estudio se incluyen variantes del SK distintas a la epidémica, la incidencia global del SK en nuestro centro fue 1,40 por 100 pacientes/año, cifra similar a la reportada por Lasso et al.

El SK clásico tiende a ser una condición crónica. Su curso clínico puede ser indolente o evolucionar con compromiso ganglionar o visceral, afectando seriamente la calidad de vida. $\mathrm{Su}$ tratamiento puede ser local o sistémico ${ }^{14,15,20}$.

En nuestro país, el único dato publicado acerca de esta variedad ha sido aportado por el estudio realizado en el Hospital Clínico de la Universidad de Chile, que incluyó 12 pacientes con biopsias compatibles con SK. Tras la revisión de las biopsias, se confirmó el diagnóstico de SK solo en 7 pacientes, 3 de ellos presentaban la variedad clásica ${ }^{6}$.

En cuanto al tratamiento con RT, Caccialanza et al. trataron 711 lesiones de SK clásico con dosis entre 10 y $40 \mathrm{~Gy}$ (dosis media: 29,24 Gy, 2-5 Gy/fracción) con lo que consiguieron una tasa de curación de $98,7 \%$, sin que se registraran efectos adversos. Nuestros resultados concuerdan con los publicados en la literatura, dado que las dosis administradas a los pacientes incluidos en el presente estudio fueron efectivas para alcanzar la respuesta clínica completa de las lesiones y el alivio sintomático, sin que se presentaran efectos adversos graves durante el tratamiento ni recidiva local durante el seguimiento ${ }^{15,21}$.

En el presente estudio todos los pacientes de este grupo presentaron enfermedad exclusivamente cutánea y no extensa, por lo que parece razonable plantear el tratamiento local en enfermedad exclusivamente cutánea, reservando la QT a las formas agresivas y difusas, sobre todo considerando los efectos adversos potenciales del tratamiento citotóxico en pacientes añosos o frágiles $^{19,22}$. En pacientes con enfermedad indolente, algunos autores han propuesto que la vigilancia activa es una buena alternativa ${ }^{23}$.

El SK iatrogénico ha sido reportado en pacientes con inmunosupresión farmacológica, tanto en aquellos con enfermedades sistémicas como en pacientes trasplantados. La frecuencia del SK en pacientes trasplantados oscila entre 1,2 y $8 \%$, 
mientras que en pacientes con inmunosupresión farmacológica por otras causas, su incidencia sería 2-4 veces menor ${ }^{15,25,26}$. Aunque puede tener un curso agresivo y compromiso visceral frecuente, ninguno de los pacientes incluidos en este estudio presentó esta evolución. En Chile no hay datos publicados respecto de su incidencia ni de su tratamiento.

Se ha descrito que la disminución de la dosis de inmunosupresores, el cambio de tratamiento o la suspensión de este pueden causar la regresión de la enfermedad ${ }^{26}$. La experiencia recogida en la literatura acerca del uso de la RT como tratamiento local en pacientes con SK iatrogénico es escasa y no se han establecido dosis ni fraccionamientos estándar. En nuestra experiencia, los esquemas de dosis utilizados fueron eficaces, seguros, bien tolerados y no conllevaron morbilidad importante. Las buenas respuestas al tratamiento obtenidas en el presente estudio son concordantes con los casos publicados en la literatura, por lo que la RT puede ser considerada como una buena opción en estos pacientes ${ }^{15}$.

Sin duda, la mayor parte de la atención ha sido puesta en la forma epidémica del SK. Es bien sabido que su evolución puede ser más agresiva que las otras variantes, comprometiendo vísceras, linfonodos o sitios inusualmente afectados por las otras variedades de $\mathrm{SK}^{7,27}$.

Según datos internacionales, la introducción de la TAAGA influyó determinantemente en el descenso de la incidencia del SK epidémico, por lo que su tratamiento actual está basado en TAAGA asociada o no a QT. La TAAGA produce respuestas completas y prolongadas en un elevado número de pacientes $^{12}$. La doxorrubicina liposomal es generalmente utilizada como QT de primera línea, dada su buena actividad sobre el SK y su favorable perfil de toxicidad ${ }^{13}$. En nuestro medio, Lasso et al. reportaron remisiones completas en $46 \%$ de los pacientes y respuestas parciales en $16 \%$ cuando se realizó tratamiento con TAAGA asociado o no a QT con bleomicina y vincristina ${ }^{4}$.

En el presente estudio, $87 \%$ de pacientes se encontraban en tratamiento con TAAGA, pese a lo cual presentaban lesiones sintomáticas y solo $33 \%$ recibió tratamiento asociado con quimioterapia. Es necesario recalcar que nuestra serie incluye pacientes tratados desde mediados de la década 1990-99, momento en el que el acceso a estos tratamientos era limitado, pues el acceso universal de garantías explícitas (AUGE) incluyó el diagnóstico y tratamiento del VIH desde el año $2006^{7,28}$.

En el contexto del SK epidémico, el rol paliativo la RT ha sido demostrado ampliamente dado su efecto antiálgico, hemostático y antidematoso ${ }^{14}$. Cabe destacar que, en nuestra serie, un buen número de pacientes presentaba dolor y edema del área afectada, mientras que el sangrado lesional estuvo presente en un número considerable de casos.

El tejido óseo es un sitio inusualmente afectado por el SK, aun cuando se presenta mayormente en asociación con la variedad epidémica, también se ha descrito en los otros tipos. El esqueleto axial es el más frecuentemente comprometido ${ }^{27}$. El compromiso óseo es un factor de pronóstico ominoso y la RT ha sido un tratamiento eficaz para la paliación de síntomas en los casos publicados en la literatura. Al momento del diagnóstico de las lesiones óseas, ambos pacientes incluidos en el presente estudio presentaban enfermedad cutáneo-visceral extensa, sin respuesta a la TAAGA asociada a QT con ciclofosfamida en uno de ellos y doxorrubicina liposomal en el segundo caso. La afectación fue del esqueleto axial (vertebral y pelviano) y el tratamiento con RT produjo respuesta completa de los síntomas.

Respecto de las dosis de tratamiento en la variedad epidémica del SK, Stelzer et al. demostraron una correlación entre la dosis administrada y la respuesta tumoral en un ensayo en el que los pacientes fueron distribuidos en forma aleatoria a un tratamiento de 8 Gy en 1 fracción versus 40 Gy en 4 semanas. Dosis mayores de RT se asociaron a mayor tasa de respuesta, menor incidencia de hiperpigmentación residual y mayor control tumoral en el tiempo. Sin embargo, la dosis de 8 Gy en 1 fracción fue una buena alternativa para la paliación de lesiones en pacientes con mal estado general, enfermedad avanzada y corta expectativa de supervivencia. Los resultados obtenidos en el presente trabajo en cuanto a la respuesta clínica tumoral y alivio sintomático son similares a los reportados en la literatura ${ }^{16,17,21}$.

Las limitaciones de nuestro estudio son su diseño retrospectivo y el reducido número de pacientes incluidos, lo que condiciona importantes dificultades para la comparación entre grupos de tratamiento, sobre todo considerando la baja frecuencia del problema estudiado. Además, el seguimiento no fue suficientemente 
extenso para el registro de resultados cosméticos a largo plazo, toxicidades tardías ni de recidivas. No obstante, su aporte radica en contribuir a los datos epidemiológicos disponibles a día de hoy en nuestro país, considerando las falencias existentes en Chile respecto a los registros de cáncer. Estas falencias podrían determinar una subvaloración del problema.

Adicionalmente, para nuestro conocimiento, es el primer estudio que recoge datos relativos al tratamiento de las variedades no epidémicas y que analiza los resultados de la RT en nuestro medio, proponiendo este tratamiento como una alternativa razonable para las distintas variedades clínicas de esta neoplasia.

\section{Referencias}

1. Kaposi M. Idiopathisches multiples Pigmentsarkom der Haut. Arch Dermatol Syphilol 1872; 4: 265.

2. Beral V, Peterman TA, Berkelman RL, Jaffe HW. Kaposi's sarcoma among persons with AIDS: a sexually transmitted infection? Lancet 2000; 335: 123-8.

3. Stribling J, Weitzner S, Smith GV. Kaposi's sarcoma in renal allograft recipients. Cancer 1978; 42: 442-6.

4. Taylor JF, Templeton AC, Vogel CL, Ziegler JL, Kyalwazi SK. Kaposi's sarcoma in Uganda: a clinico-pathological study. Int J Cancer 1971; 8: 122-35.

5. Primer informe de registros poblacionales de cáncer en Chile. Quinquenio 2003-2007. Unidad de vigilancia de enfermedades no transmisibles y estudios. Ministerio de Salud Chile. Disponible en www.paho.org/chi [Consultado el 4 de abril de 2017].

6. Moncayo E, Wageman E, Zemelman V, Rohmann I. Sarcoma de Kaposi: a propósito de una revisión Clinicopatológica. Dermatol. Chile 1994; 10 (4): 247-25.

7. Figueroa F, Palacios A, Rivero S, Oddo D, Roa I, Honeyman J, et al. [Chronic diarrhea due to Isospora belli and Kaposi's sarcoma in a male homosexual. Report of the 1st case of acquired immunodeficiency syndrome in Chile]. Rev Med Chile 1985; 113 (8): 772-9.

8. Lasso BM, Pérez GJ, Noriega R, Malebrán RA, Espinoza VS. Sarcoma de Kaposi y VIH: Tratamiento antirretroviral y quimioterapia en 32 pacientes. Rev Med Chile 2003; 131 (5): 483-90.

9. Willatt J, Moyano C, Apey C, Lidid L. Sarcoma de Kaposi extratorácico: Evidencias de una enfermedad multisistémica. Rev Chil de Radiol 2010; 16 (2): 80-5.

10. Salvo A, Garrido J, Madrid P, González S, Porras N. Sarcoma de Kaposi en mucosas en pacientes PPVI. A propósito de 2 casos. Revisión de bibliografía. Rev Chilena Dermatol 2014; 30 (2): 184-88.

11. Chernilo S, Trujillo S, Kahn M, Paredes M, Echevarría G, Sepúlveda C. [Lung diseases among HIV infected patients admitted to the "Instituto Nacional del Tórax" in Santiago, Chile]. Rev Med Chile 2005; 133 (5): 517 24.

12. La Ferla L, Pinzone MR, Nunnari G, Martellotta F, Lleshi A, Tirelli U, et al. Kaposi' s sarcoma in HIV-positive patients: the state of art in the HAART-era. Eur Rev Med Pharmacol Sci 2013; 17 (17): 2354-65.

13. Gill PS, Wernz J, Scadden DT, Cohen P, Mukwaya GM, von Roenn JH, et al. Randomized phase III trial of liposomal daunorubicin versus doxorubicin, bleomycin, and vincristine in AIDS-related Kaposi's sarcoma. J Clin Oncol 1996; 14 (8): 2353-64.

14. Cooper JS, Steinfeld AD, Lerch I. Intentions and outcomes in the radiotherapeutic management of epidemic Kaposi's sarcoma. Int J Radiat Oncol Biol Phys 1991; 20 (3): 419-22.

15. Jakob L, Metzler G, Chen KM, Garbe C. Non-AIDS associated Kaposi's sarcoma: clinical features and treatment outcome. PLoS One 2011; 6 (4): e18397.

16. Stelzer KJ, Griffin TW. A randomized prospective trial of radiation therapy for AIDS-associated Kaposi's sarcoma. Int J Radiat Oncol Biol Phys 1993; 27 (5): 1057-61.

17. Harrison M, Harrington KJ, Tomlinson DR, Stewart JS. Response and Cosmetic outcome of two fractionation regimens for AIDS-related Kaposi's sarcoma. Radiother Oncol 1998; 46 (1): 23-8.

18. Cox JD, Stetz J, Pajak TF. Toxicity criteria of the Radiation Therapy Oncology Group (RTOG) and the European Organization for Research and Treatment of Cancer (EORTC) Int J Radiat Oncol Biol Phys 1995; 30 (31): 1341-6.

19. Brambilla L, Labianca R, Boneschi V, Fossati S, Dallavalle G, Finzi AF, et al. Mediterranean Kaposi's sarcoma in the elderly. A randomized study of oral etoposide versus vinblastine. Cancer 1994; 74 (10): 2873-8.

20. Brambilla L, Bellinvia M, Tourlaki A, Scoppio B, Gaiani $\mathrm{F}$, Boneschi V. Intralesional vincristine as first-line therapy for nodular lesions in classic Kaposi sarcoma: a prospective study in 151 patients. Br J Dermatol 2010; 162 (4): 854-9.

21. Caccialanza M, Marca S, Piccinno R, Eulisse G. Radiotherapy of classic and human immunodeficiency virus-related Kaposi's sarcoma: results in 1482 lesions. J Eur Acad Dermatol Venereol 2008; 22 (3): 297-302.

22. Brambilla L, Recalcati S, Tourlaki A. Vinorelbine thera- 
py in classic Kaposi's sarcoma: a retrospective study of 20 patients. Eur J Dermatol 2015; 25 (6): 535-8.

23. Vincenzi B, D’Onofrio L, Frezza AM, Grasso RF, Fausti V, Santini D, et al. Classic Kaposi Sarcoma: to treat or not to treat? BMC Res Notes 2015; 10 (8): 138.

24. Louthrenoo W, Kasitanon N, Mahanuphab P, Bhoopat L, Thongprasert S. Kaposi's sarcoma in rheumatic diseases. Semin Arthritis Rheum 2003; 32 (5): 326-33.

25. Zavos G, Moris D, Vernadakis S, Bokos J, Lionaki S, Mamarelis $G$, et al. Incidence and management of
Kaposi sarcoma in renal transplant recipients: the Greek experience. Transplant Proc 2014; 46 (9): 3199-202.

26. Moosa MR. Kaposi's sarcoma in kidney transplant recipients: a 23-year experience. QJM 2005; 98 (3): 205-14.

27. Pantanowitz L, Dezube BJ. Kaposi sarcoma in unusual locations. BMC Cancer 2008; 8: 190.

28. Wolff MR, Álvarez PP, Flores IS, Northland RA, Wolff CL. Evolución de mortalidad y estado actual de una población infectada por VIH controlada en un centro multiprofesional. Rev Med Chile 2006; 134: 581-8. 